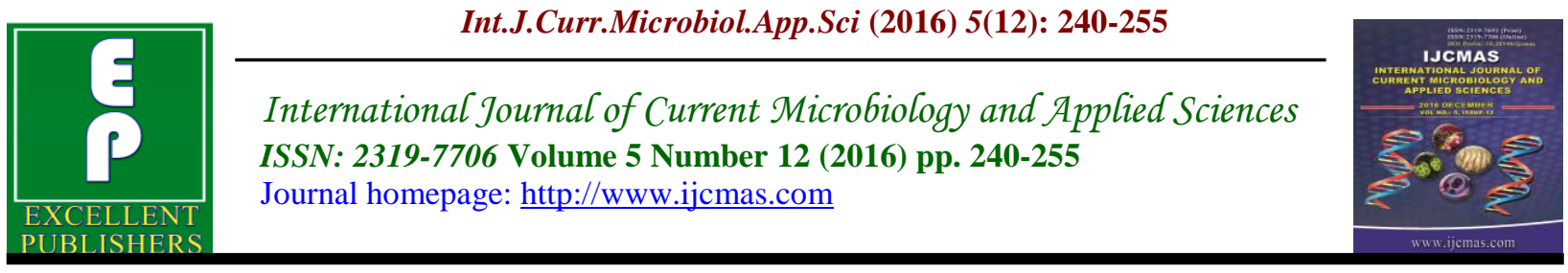

Original Research Article

http://dx.doi.org/10.20546/ijcmas.2016.512.026

\title{
Curcumin Boosts up the Efficacy of Imatinib Mesylate in Chronic Myelogenic Leukemia Cell Line K-562 by Modulation of Various Markers
}

\author{
A. Mukherjee ${ }^{1}$, R. Sarkar ${ }^{1}$, S. Mukherjee ${ }^{1}$, J. Biswas ${ }^{2}$ and M. Roy ${ }^{1 *}$ \\ ${ }^{1}$ Department of Environmental Carcinogenesis \& Toxicology, ${ }^{2}$ Director \\ Chittaranjan National Cancer Institute, 37, S P Mukherjee Road, Kolkata 700 026, India \\ *Corresponding author
}

\section{Keywords}

Chronic myelogenous leukemia, imatinib mesylate, curcumin, cytokines, Bcr-Abl, apoptosis.

\section{Article Info}

Accepted:

12 November 2016

Available Online:

10 December 2016

\section{A B S T R A C T}

Imatinib mesylate(IM) is the most commonly used drug for therapy of chronic myeloid leukemia (CML). It exerts its effect by targeting a constitutively activated tyrosine kinase Bcr-Abl, a characteristic feature of CML. This anti-tumor drug also influences various other proteins like cytokines, transcription factors and their associated signalling pathways that contribute to the development of leukemogenesis. However, it poses serious side effects. Thus attention should be given to non-toxic means of leukemia control. Phytochemicals like curcumin possess a plethora of anti-cancer properties, show minimal toxicity and may therefore come to the rescue. The present study aims to explore the potential of curcumin to boost up the efficacy of IM, and to minimize the drug dose and associated toxicity in human chronic myelogenous leukemia cell line K-562. The drug IM was found to modulate the expression of markers deregulated in CML. However, curcumin refines the efficacy of IM, especially when added prior to drug treatment. Similar trend was reflected in induction of programmed cell death. Thus the phytochemical curcumin may enhance the potency of IM used in leukemia therapy, thereby suggesting its role in an adjuvant therapy for CML.

\section{Introduction}

Leukemia, a type of malignancy, originates in the bone marrow and results in increased proliferation of abnormal white blood cells. Leukemia may be acute or chronic, and various types have been identified (Udensi and Tchounwou, 2014). Among different types, chronic myelogenous leukemia (CML) is the most prevalent form in Asian countries (Anand et al., 2012), which may be divided into three phases, namely an initial chronic phase followed by accelerated and blastic phases (Valent, 2007).
The Philadelphia chromosome, resulting from a reciprocal translocation between the long arms of chromosomes 9 and 22, is a very common feature of CML. It has constitutive tyrosine kinase activity (Anand et al., 2012). There are some endogenous proteins or metabolites called biomarkers, which are aberrantly expressed in cancer and are hence considered as predictive indicators. A group of cytokines, regulating proliferation of blast cells are biological markers in leukemia. Tumor necrosis factor 
alpha (TNF $\alpha$ ), belonging to the TNF/TNFR cytokine superfamily is an important mediator of inflammation and associated disorders including cancer (Wang and Lin, 2008). TNF $\alpha$, on the other hand activates Nuclear factor kappa B (NF-kB), which is a transcription factor mediating antiapoptotic signals in malignancy. NF- $\kappa \mathrm{B}$, consisting of five subunits normally resides in the cytoplasm, which upon phosphorylation and subsequent ubiquitination and proteasomal degradation of $\mathrm{I} \kappa \mathrm{B}$ translocates to the nucleus, where it is active. p50 and p65 are the two most important subunits of NF-kB (Lawrence, 2009). Cytokines may be pro and anti-inflammatory. NF-kB regulates the pro-inflammatory cytokine interleukin- 8 (IL-8) which plays an important role in cell proliferation and survival (Elliott et al., 2001). Production of IL-8 is known to be increased by various stimuli including TNFa. IL-10, a multifunctional antiinflammatory cytokine is also transcriptionally controlled by $\mathrm{NF}-\kappa \mathrm{B}$ (Driessler et al., 2004). Cancer cells encounter various forms of stresses, including oxidative stress, due to which an important group of proteins called Heat Shock Proteins (HSPs) get induced (Nahleh et al., 2012). Of all types of HSPs, HSP 90 is a major regulator of signal transduction and cellular proliferation and it is abundantly expressed in leukemia (Flandrin et al., 2008). Furthermore HSP 90 plays an important role in $\mathrm{NF}-\kappa \mathrm{B}$ mediated inhibition of apoptosis (Lewis et al., 2000; Sarkar et al., 2014). Survivin, a member of the inhibitor of apoptosis protein family (IAP) plays a pivotal role in carcinogenesis by suppressing apoptosis and promoting cell division (Kelly et al., 2011). TNF- $\alpha$ upregulates the expression of survivin via $\mathrm{NF} \kappa \mathrm{B}$ activated signaling cascade (Ahmeda et al., 2012). All these markers may be considered as potent targets in leukemia therapy.
During the last decade, there has been a considerable advancement in CML therapy, leading to better prognosis. Commonly used treatment modalities for CML are chemotherapy (busulfan and hydroxyurea etc), targeted therapy (Imatinib Mesylate etc), immunotherapy, radiotherapy and bone marrow transplantation (Henkes et al., 2008). Imatinib Mesylate (IM) nowadays is considered to be the standard therapy of myeloid leukemia which works by targeting these biomarkers aberrantly expressed in leukemia and is also a specific inhibitor of the Bcr-Abl tyrosine kinase activity (An et al., 2010).

However IM shows severe side effects like higher susceptibility to infections, skin rash, fatigue, muscle cramps, and diarrhea. Apart from these, IM is also reported to be myelosuppressive, hepatotoxic and nephrotoxic (Henkes et al., 2008). To overcome the problem of IM toxicity, it is desirable to reduce the drug dose so that the associated toxicity can be minimized. The anticancer drugs target actively proliferating cells in general, they cannot discriminate the normal healthy cells from the cancerous ones. Therefore, besides killing the cancer cells, the healthy cells are also damaged leading to adverse side effects (Singh et al., 2008). There are certain plant derived molecules that can destroy cancer cells, sparing the normal ones. These molecules, coined as phytochemicals may have some differential effect on cancer cells and act as chemo-enhancer (Gopalakrishnan and Tony kong, 2008; Sak, 2012). The golden spice Curcumin, obtained from the rhizomes of the plant Curcuma longa may be helpful in this regard as it possesses antioxidant, chemo-preventive, chemotherapeutic, and chemo-sensitizing activities and it acts by targeting some of the key molecules involved in the carcinogenesis (Jiao et al., 2009). Curcumin though bestowed with 
various disease healing properties, have poor bioavailability; another plant ingredient piperine has been found to take care of this problem (Suresh and Srinivasan, 2010). Present study aims to assess the potential of curcumin in sensitizing the leukemic cells towards the anti-cancer drug IM. This study may shed some light on the putative role of Curcumin as a chemo-enhancer in leukemia by targeting certain biomarkers involved in the process of leukemogenesis.

\section{Materials and Methods}

Cell culture media RPMI-1640 was procured from GIBCO-BRL India Pvt. Ltd, New Delhi, India. Acrylamide, N, N'methylenebisacrylamide, fetal bovine serum (FBS) and ELISA kits to detect the levels of cytokines TNF- $\alpha$, IL- 8 and IL-10 were purchased from Invitrogen BioServices India Pvt. Ltd., Bangalore, India. Phytohaemagglutinin (PHA) was obtained from GIBCO-BRL India Pvt. Ltd, New Delhi, India. Histopaque, dithiothreitol (DTT), bovine serum albumin (BSA), Ponceau S, ethylene glycol-O,-O'-bis, (2aminoethyl) N,N,N',N'-tetra acetic acid (EGTA), 4-(2-hydroxyethyl)-1piperazineethanesulfonic acid (HEPES), CHAPS, RNase A, proteinase $\mathrm{K}$, IM and propidium iodide $(\mathrm{PI})$ were procured from Sigma Chemical Co, St. Louis, MO, USA. Curcumin was obtained from SigmaAldrich, St Louis, MO, USA. Goat antimouse IgG-alkaline phosphatase conjugate and 5-bromo-4-chloro-3-indolyl phosphate/ nitro blue tetrazolium (BCIP-NBT) were obtained from Bangalore Genei, India. Nitrocellulose membrane was procured from Hybond ECL, Amersham Biosciences, UK. Tris, sodium dodecyl sulfate (SDS) and glycine were obtained from Amresco, Ohio, USA. Assay kits from Millipore, Billerica, MA were used to detect activities of caspases 3, 8 and 9. Antibodies against TNF- $\alpha$ and survivin (Biorbyt), IL- 8 and IL-
10 (Abcam), NF- $\kappa \mathrm{B}(\mathrm{p} 50)$ and $\mathrm{NF}-\kappa \mathrm{B}(\mathrm{p} 65)$ and HSP90 (Santa Cruz) were used for western blotting technique. Anti-BCR-ABL Monoclonal Antibody [7C6] (MA1-153) was purchased from Pierce; Thermo Scientific, USA. Other reagents of analytical grade are purchased locally. The instruments used were Gel doc apparatus (BioRad), fluorescence microscope (Leica), spectrofluorimeter (Varian), ELISA plate reader (Tecan) and spectrophotometer (Varian).

\section{Methods}

\section{Cell culture}

Chronic myelogenous leukemia cell line K562 (human origin) were cultured as per laboratory protocol (Roy et al., 2015). They were grown at $37^{\circ} \mathrm{C}$ in a humidified $5 \% \mathrm{CO}_{2}$ atmosphere in RPMI 1640, with 10\% FBS and antibiotics to prevent any unwanted bacterial contamination.

\section{Isolation and culture of lymphocytes}

Lymphocytes obtained from healthy human donor were used as control for the study. They were maintained as per laboratory protocol (Sarkar et al., 2014) and seeded in RPMI-1640 supplemented with 10\% FBS, antibiotics and $20 \mu \mathrm{g} / \mathrm{ml}$ Phytohaemagglutinin (PHA). The lymphocytes thus isolated are grown in an ambient condition of $37^{\circ} \mathrm{C}$ in a humidified atmosphere of $5 \% \mathrm{CO}_{2}$.

\section{Treatment protocol}

Exponentially growing cells were treated with different concentrations of IM $(0.5,1$, 2.5 and $5 \mu \mathrm{M})$ in absence and presence of Curcumin $(30 \mu \mathrm{M})$. Different modes of treatment were followed: (I) treatment with IM alone, (II) simultaneous mode, where exponentially growing cells were treated 
with Curcumin and IM simultaneously for 24 hours and (III) pre-treatment mode, where cells were treated with Curcumin for 24 hours prior to treatment with IM for the next 24 hours.

\section{Assessment of cell cytotoxicity}

Cytotoxicity of antitumor drug IM to K-562 was measured by MTT assay. Reduction of 3-(4, 5-dimethythiazol-2-yl)-2,5-diphenyl tetrazolium bromide (MTT) by the mitochondrial dehydrogenase of live cells are measured spectrophotometrically. The colour developed, hence the OD gives an estimate of viable cells. The formazan product formed are soluble in DMSO giving a purple coloration and the absorbance was measured at $570 \mathrm{~nm}$.

\section{Enzyme-linked immunosorbent assay}

The levels of cytokines (TNF- $\alpha$, IL-8, and IL-10) were studied using ELISA kits employing a technique called quantitative sandwich immunoassay. Samples were added to microtiter plate pre-coated with an antibody specific to the interleukins along with a biotin-conjugated antibody preparation and incubated as prescribed. In order to determine the amount of the specific interleukin present, the sample was incubated with avidin conjugated Horseradish Peroxidase (HRP) and a TMB (3, 3', 5, 5' tetramethyl-benzidine) substrate solution was added. The colour change was measured spectrophotometrically at a wavelength of $450 \mathrm{~nm}$.

\section{Western blotting analysis}

Expression of NF- $\kappa \mathrm{B} \quad(\mathrm{p} 50$ and p65 subunits), survivin, HSP90 and p210 ${ }^{\mathrm{Bcr}-\mathrm{Abl}}$, were assessed by Western blot (WB) analysis using corresponding antibodies, following the protocol of Sarkar et al (Sarkar et al., 2013). $\beta$-actin and TBP were used as loading control.

\section{Assessment of apoptosis by staining with PI}

Treated cells were harvested, washed with PBS, stained with Propidium Iodide solution $(50 \mu \mathrm{g} / \mathrm{ml})$ and incubated for $10 \mathrm{~min}$ in the dark at room temperature. Cells taken on a glass slide were visualized under a fluorescence microscope (Leica) for assessment of apoptotic features.

\section{Assessment of caspase activity}

Treated cells were harvested. Cell lysates were prepared to assess the activities of caspases 3, 8 and 9 as per manufacturer's protocol. These assays employ synthetic substrates DEVD-AFC, IETD-AFC and LEHD-AFC respectively for caspases 3,8 and 9. A fluorophore AFC (7-amino-4trifluoromethyl coumarin) is tagged with these synthetic peptides. Caspases release free AFC upon cleavage of the synthetic substrate, which emits a yellow green fluorescence at $480-520 \mathrm{~nm}$ (peak at 505 $\mathrm{nm})$ upon excitation at $400 \mathrm{~nm}$. The fluorescence intensity was estimated by a spectrofluorimeter (Varian).

\section{DNA Fragmentation}

Extraction of fragmented DNA was done following the protocol of Hermann et al (Hermann and Frischauf, 1987). Treated cells were harvested, washed and collected by centrifugation. Then cells were exposed to lysis buffer and centrifuged. 1\% SDS was then added to the collected supernatant. The mixture was incubated overnight with 5 $\mu \mathrm{g} / \mathrm{ml}$ RNase A at $56^{\circ} \mathrm{C}$. The mixture was further digested with $2.5 \mu \mathrm{g} / \mathrm{ml}$ proteinase $\mathrm{K}$ for 4 hours at $37^{\circ} \mathrm{C}$. 0.5 volume of $10 \mathrm{M}$ ammonium acetate was added and DNA was precipitated using 2.5 volume of cold ethanol. The extracted DNA was air-dried and resuspended in loading buffer and 
resolved on $1.5 \%$ agarose gel. Fragments of DNA were visualized using Gel doc apparatus (BioRad).

\section{PARP degradation}

Immunocytochemistry was performed to determine PARP degradation by caspases during apoptosis. Treated cells were harvested, seeded on coverslips; air dried and fixed using formaldehyde solution. They were then permeabilized with ice-cold acetone and blocked with BSA. The cells were incubated with anti-PARP monoclonal primary antibody, washed with PBS and incubated with FITC conjugated secondary antibody at $37^{\circ} \mathrm{C}$ in a dark humid chamber. The cells were viewed under a fluorescence microscope and photographed.

\section{Statistical Analysis}

SPSS 10.0 (one way ANOVA followed by Dunett t-test) was used for statistical anlysis.

\section{Results and Discussion}

\section{Determination of toxicity of IM and Curcumin}

MTT assay has been carried out to assess the cytotoxicity of IM and Curcumin towards K562 cells and peripheral blood lymphocytes (PBL) isolated from healthy donor. Results reveal that IM is toxic to both the cells, though the extent of killing is more in K-562 than PBL (Fig 1A). On the other hand, Curcumin shows its cytotoxic effect towards K-562 cells only; very little toxicity towards PBL has been noticed at the higher concentrations (Fig 1B). As shown in the graph, only $20 \% \mathrm{~K}-562$ cells survive when treated with $50 \mu \mathrm{M}$ Curcumin for 24 hours, therefore for all subsequent experiments, 30 $\mu \mathrm{M}$ has been chosen as the highest dose of Curcumin where $50 \%$ cell killing is achieved.

\section{Aberrant expressions of tumor markers in $\mathrm{K}-562$ cells}

Western blot analysis has been performed to assess the constitutive expression of tumor markers cytokines (TNF- $\alpha$, IL-8, IL-10), NF- $\kappa$ B (p50 \& p65 subunits), survivin, HSP 90 and p210 ${ }^{\mathrm{BCR}-\mathrm{ABL}}$ in leukemic cells K-562 and PBL isolated from healthy donor. Results reveal that cytokines (TNF- $\alpha$, IL-8), NF- $\kappa \mathrm{B}$ (p50 and p65), survivin, HSP 90 and $\mathrm{p} 210^{\mathrm{Bcr}-\mathrm{Abl}}$ are constitutively high in K-562 cells compared to the PBL. Expression of anti-inflammatory cytokine IL-10 however is low in the leukemia cells than normal lymphocytes (Fig 2).

\section{Modulation of cytokines and other tumor markers by IM in absence and presence of Curcumin}

K-562 cells have been treated with increasing concentrations of IM $(0,0.5,1$, $2.5,5 \mu \mathrm{M})$ for 24 hours, either alone or in conjunction with $30 \mu \mathrm{M}$ Curcumin. ELISA has been carried out to assess the levels of cytokines in these cells. Pro-inflammatory cytokines, TNF- $\alpha$ (Fig 3A) and IL-8 (Fig 3B) are found to be downregulated dosedependently by IM both in absence and presence of $30 \mu \mathrm{M}$ Curcumin. IM hardly shows any effect on the levels of IL-10. However, in presence of curcumin, the marker has been found to be up-regulated (Fig 3C). Western blot analysis has been performed to investigate the modulation of other leukemic markers by IM in absence and presence of $30 \mu \mathrm{M}$ Curcumin. Transcription factor $\mathrm{NF}-\kappa \mathrm{B}$ subunits $\mathrm{p} 50$ and p65, survivin, HSP90 and p210 ${ }^{\mathrm{Bcr}-\mathrm{Abl}}$ are found to be negatively regulated by the drug IM alone as well as when used in the combinatorial treatment modality. The corresponding band intensities are given in the bar diagram and fold change have been calculated based on band intensity values, 
which represent the mean from three independent experiments (Fig 4). The fold change of leukemic biomarkers achieved by treatment with curcumin has been tabulated in Table 1.

\section{Induction of programmed cell death in leukemia cells}

Treatment of cells with a combination of IM and Curcumin has been found to induce apoptosis as evident from fluorescence microscopic examination of morphological features of the cells. Representative micrographs show presence of typical features of apoptosis, namely shrinkage of cells, condensation of chromatin, fragmented nuclei, membrane blebbing and formation of apoptotic bodies (Fig 5A). The apoptotic index (i.e the ratio of the apoptotic to the non apoptotic cells) has been calculated and are depicted in the bar diagram (Fig 5B). Apoptotic indices are higher in presence of curcumin and that too in pretreatment modality.

\section{Activation of caspases 3, 8 and 9}

Caspases, a group of cysteine-dependent aspartate-directed proteases serve as key regulators of the apoptotic pathway. Cells are treated as described before and the activities of caspases 3,8 and 9 have been assessed by fluorimetric analysis. Results show that IM increases the activity of caspases in K-562; presence of curcumin along with IM enhances the effect further, especially in the pre-treatment modality (Fig 6A).

It is clear from the results that fold increase in caspase 3 and 9 are maximum (data not shown) compared to caspase 8, indicating that induction of programmed cell death is facilitated mainly by the intrinsic pathway.

\section{Study of DNA Fragmentation, a hallmark of apoptosis}

DNA fragmentation is a characteristic hallmark of apoptosis. DNA fragmentation is assessed by subjecting extracted DNA to gel electrophoresis. During apoptosis, activated endonucleases cleave chromatin DNA into 180 bp nucleosomal unit fragments or multiple of that. Characteristic fragmentation of DNA has been depicted in Fig 6B, showing that laddering, which is an indicator of internucleosomal fragments is maximum when cells are treated with IM and curcumin in pretreatment modality. Results are at par with those from apoptotic index values.

\section{Degradation of PARP}

K-562 cells, after treatment with either IM, alone or in combination with Curcumin are incubated with anti-PARP antibody and labeled with FITC conjugated secondary antibody. The cells have been viewed under a fluorescence microscope and representative photographs are given in Fig 6C. Expression of PARP is higher in untreated K-562 cells as evident from the fluorescent intensity. The intensity has been found to be diminished when treated with IM; extent of decrease in fluoresence intensity is more in presence of curcumin and that is even higher in pre-treatment modality, indicating degradation of PARP.

Several proteins called tumor markers are aberrantly expressed in cancer and their deregulation leads to carcinogenesis. Treatment strategy is based on targeting these markers (Magee et al., 2013). Drugs, used in the treatment of cancer often show severe toxicity and associated side effects (Naidu et al., 2004), though they differ in their mechanism of action and impact on an individual. Apart from their desired action 
on cancer cells, anticancer drugs often cause severe and sometimes painful side effects. It would have been the ideal situation if the anticancer drugs could bind only to the specific proteins they are meant to target, but, in reality, in addition to these proteins, they exert their action on other proteins as well.

This is a problem in cancer therapy. Imatinib Mesylate is a drug of choice for the treatment of leukemia, which besides targeting leukemia cells, also harm the normal healthy cells (Mughal and Schrieber, 2010). This poses serious concern to control leukemogenesis, by making an individual sick and leading to poor prognosis. Therefore, non-toxic means of leukemia control needs considerable attention. Curcumin, a phytochemical with potent anticancer properties may be helpful in this context (Tuorkey, 2014). This plant derived molecule may be employed with a curative intent, so that the toxicity of antitumor drugs, hence side effects may be minimized. These phytochemicals can aid in reducing the drug dose. Curcumin showed minimal toxicity in peripheral blood lymphocytes isolated from normal healthy individuals, but, is potent to modulate the target proteins, aberrantly expressed in chronic myelogenic leukemia cell K-562. Through amelioration of target proteins, curcumin makes the cells vulnerable to killing by anticancer drugs. Hence lesser dose of drug can achieve the same extent of cell killing in presence of plant derived molecules. In order to prove the efficacy of curcumin, it is worthwhile to understand the mechanism.

Previous findings from our laboratory showed that curcumin down-regulates HSPs with a concomitant up-regulation of proapoptotic proteins Bax, Bad, Bid, and AIF and decrease in the expression of antiapoptotic protein Bcl-2, resulting in release of cytochrome c from mitochondria and activation of caspases (Sarkar et al., 2014). The anticancer drugs work with the ultimate aim of achieving programmed cell death, but the normal healthy cells are not spared. Therefore, drug dose minimization is important in cancer therapeutics. Curcumin aids in diminution of drug dose.

Table.1 Modulation of leukemic markers by IM, as influenced by curcumin

\begin{tabular}{|c|c|c|c|}
\hline \multirow[t]{2}{*}{ Marker } & \multicolumn{3}{|c|}{ Fold change } \\
\hline & I & II & III \\
\hline TNF- $\alpha$ & $\mathbf{1}$ & 0.75 & $0.47^{\mathrm{a}}$ \\
\hline IL-8 & 1 & 0.72 & $0.46^{\mathrm{a}}$ \\
\hline IL-10 & $\mathbf{1}$ & 2.19 & $3.06^{b}$ \\
\hline NF- $\kappa B$ (p50 subunit) & $\mathbf{1}$ & 0.67 & $0.19^{b}$ \\
\hline $\mathrm{NF}-\kappa \mathrm{B}(\mathrm{p} 65$ subunit) & 1 & 0.52 & $0.12^{b}$ \\
\hline Survivin $\downarrow$ & 1 & 0.48 & $0.19^{b}$ \\
\hline HSP 90 & $\mathbf{1}$ & 0.70 & $0.15^{b}$ \\
\hline p210 $\mathrm{BCR-ABL}$ & $\mathbf{1}$ & $\mathbf{0 . 5 3}$ & $0.17^{b}$ \\
\hline
\end{tabular}

Fold change in the level of markers are significantly different in the pretreatment modality (III) compared to the simultaneous modality (II). The values are significant ${ }^{a}(p<0.05),{ }^{b}(p<0.005)$ in III with respect to II. 
Fig.1 Effect of IM and Curcumin on proliferation of K-562 cells and human PBL. (A) K-562 cells and human lymphocytes (PBL) from healthy individuals were treated with IM $(0,0.5,1$, 2.5, $5 \mu \mathrm{M}$ ) for 24 hours and subjected to MTT assay. (B) K-562 cells and human lymphocytes were treated with curcumin $(0,0.5,1,10,30,50 \mu \mathrm{M})$ for 24 hours. Results from MTT assay show that IM was toxic to both the cells, though toxicity in K-562 is more, whereas curcumin is preferentially toxic to leukemia cells, not human PBL.

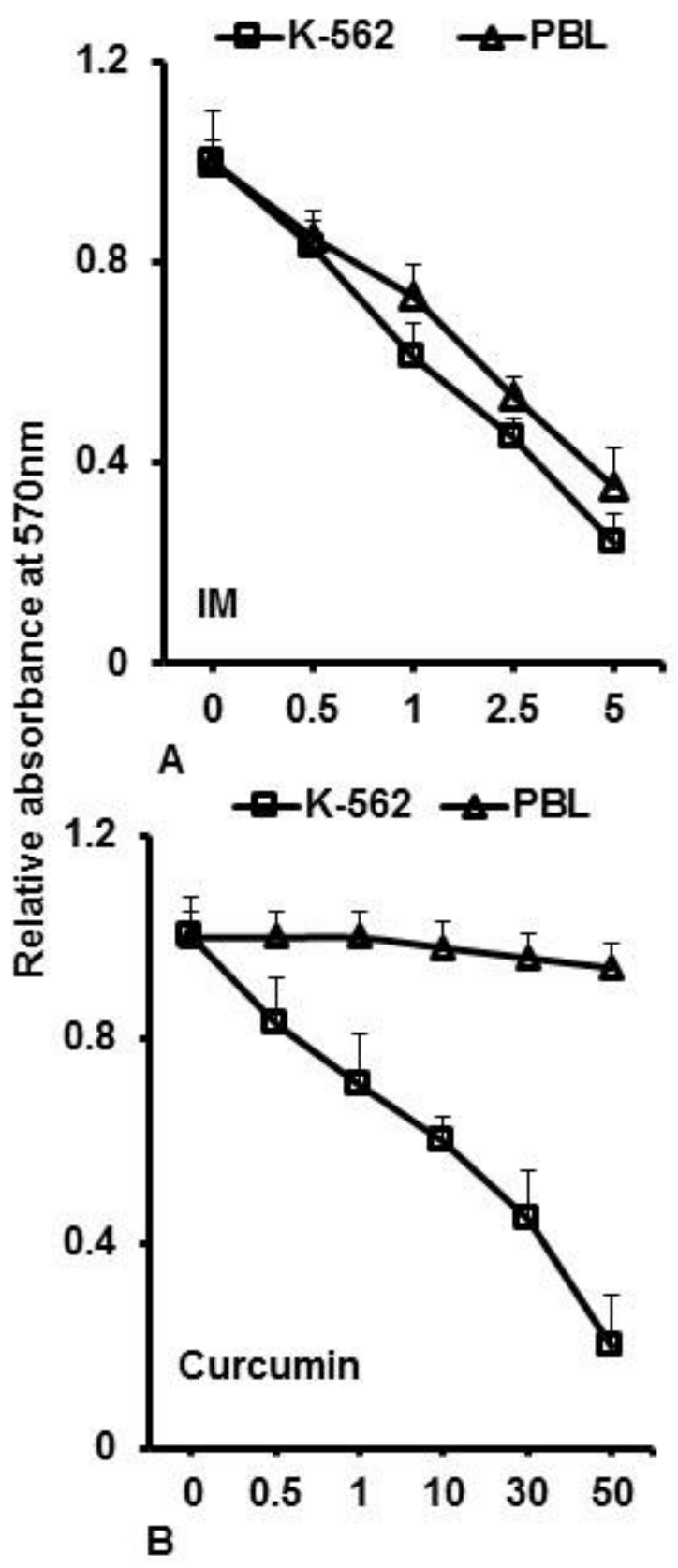

Concentration $(\mu \mathrm{M})$ 
Fig.2 Constitutive expressions of certain biomarkers. (A) Expression of TNF- $\alpha$, IL-8, IL-10, NF$\kappa \mathrm{B}$ (p50\&p65), survivin, HSP90 and p210BCR-ABL in K-562 (lane1) and PBL (lane2) were assessed by WB. TBP was the loading control for NF- $\kappa \mathrm{B}$ subunits whereas $\beta$-actin for others. (B) Expressions of biomarkers were depicted quantitatively from band intensities. Protein expressions were significantly higher ( $p$ value $<0.005$ ) in $\mathrm{K}-562$ cells as compared to normal lymphocytes, except for IL-10, where the expression in K-562 cell is significantly much lower ( $p$ value <0.005) than PBL. Each band intensity represents the mean from three independent experiments $\pm \mathrm{SE}$.

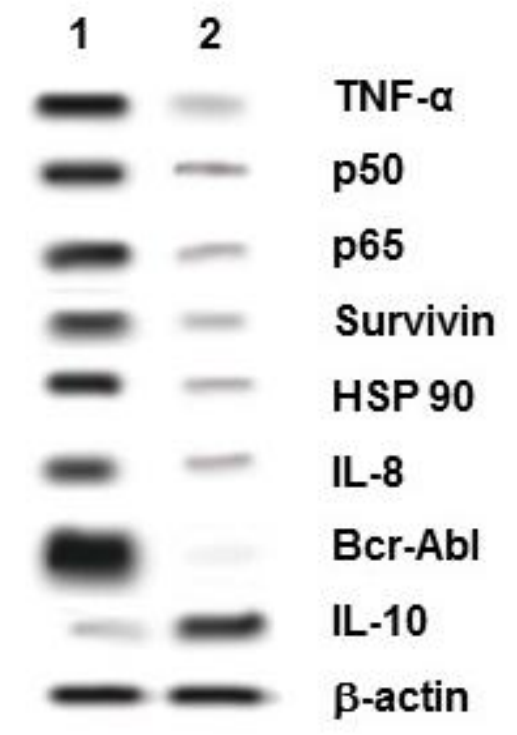

A

由 562 \&PBLN

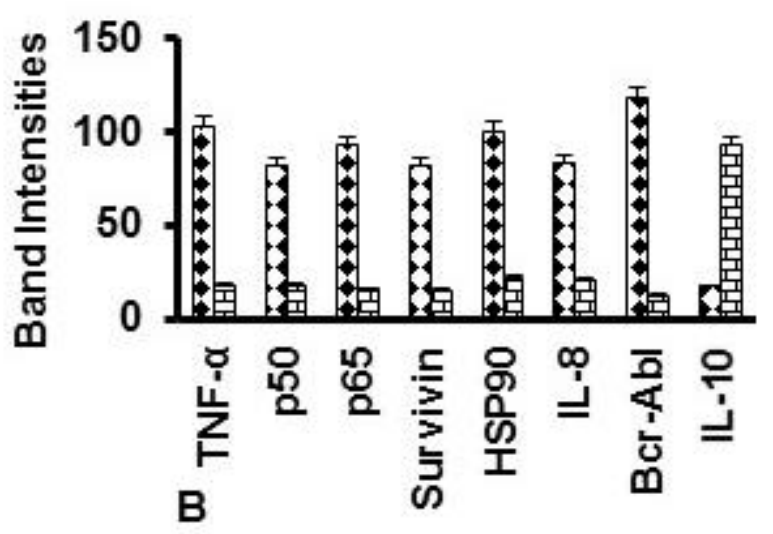


Fig.3 Effect of IM on biomarkers as influenced by Curcumin. Cells were treated with IM alone (I), along with Curcumin $(30 \mu \mathrm{M})$, both in simultaneous(II) and pre-treatment modality(III); levels of TNF- $\alpha$, IL-8, IL-10 were shown in A,B,C respectively as assessed by ELISA. TNF- $\alpha$ \& IL-8 were down-regulated by IM, alone as well as in presence of curcumin (II \& III), extent of decrease is more in the later. Levels of IL-10 however were hardly affected by IM, but presence of Curcumin increased the level, and that too is more in III. Results are mean of three independent experiments \pm SE and values are significant $\mathrm{a}(\mathrm{p}<0.05), \mathrm{b}(\mathrm{p}<0.005)$ and $\mathrm{c}(\mathrm{p}<0.0005)$ with respect to the cells treated with IM alone.
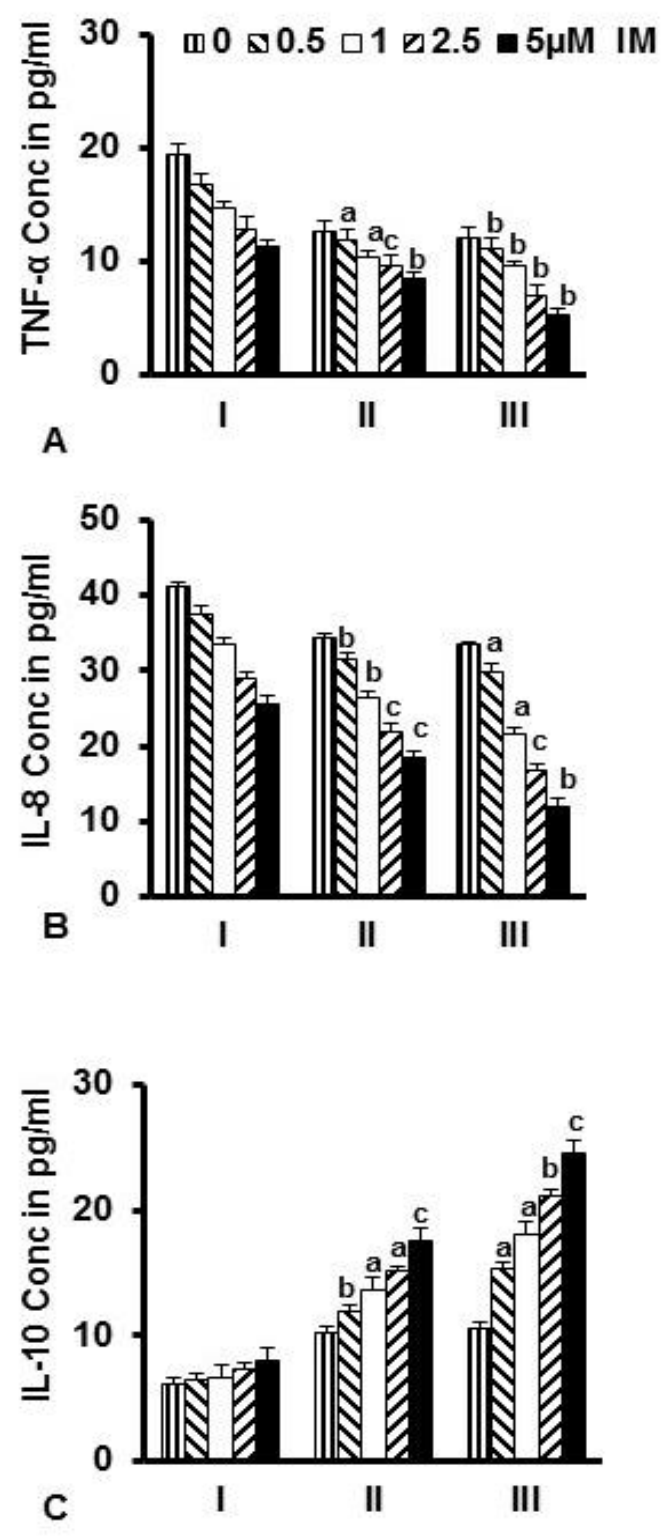
Fig.4 Effect of IM on other leukemic biomarkers as influenced by Curcumin. (A) Cells were treated with IM alone (I) and in combination with Curcumin $(30 \mu \mathrm{M})$, both in simultaneous(II) \& pre-treatment modality (III). WB was performed to assess the expressions of NF- $\mathrm{KB}$ (p50\&p65), survivin, HSP90 and p210BCR-ABL. WB bands are shown in the figure. (B) Corresponding band intensities (average of 3 independent experiments \pm SE) were shown. IM downregulated the expressions of the markers both in absence and presence of Curcumin, however, better effect was observed when cells were pre-treated with curcumin. Values are significant $\mathrm{a}(\mathrm{p}<0.05)$, $\mathrm{b}(\mathrm{p}<0.005)$ and $\mathrm{c}(\mathrm{p}<0.0005)$ with respect to only IM treated cells.

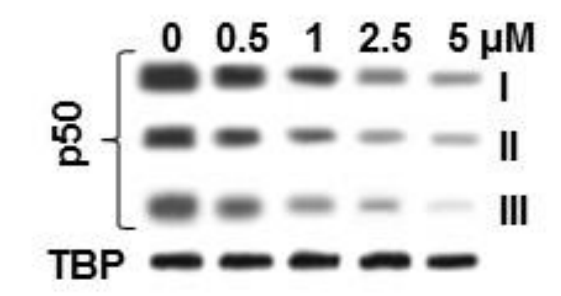

๓0 0 จ $0.5 \square 1 \nabla 2.5 \square 5 \mu \mathrm{M}$ IM
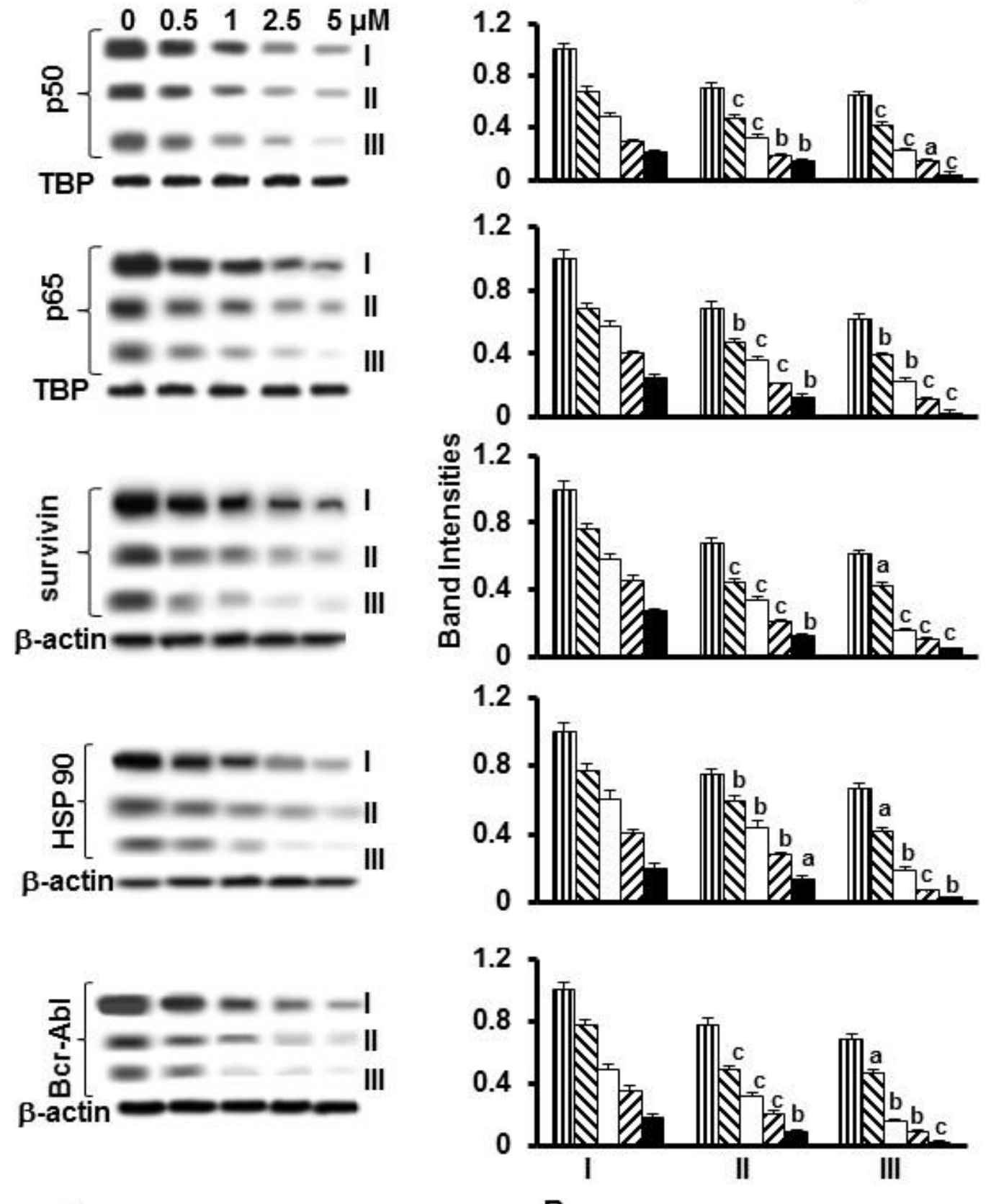

A

B 
Fig.5 Effect of IM on induction of apoptosis as influenced by Curcumin. Cells were treated with IM alone (I) and in combination with Curcumin $(30 \mu \mathrm{M})$, both in simultaneous(II) and pretreatment modality(III). (A) Images of PI stained cells are shown: (Con) shows untreated cells; (I), (II), (III) respectively represent cells after treatment with $5 \mu \mathrm{M}$ IM alone, IM with Curcumin, simultaneously and pretreated. Apoptotic features were studied under a fluorescence microscope and apoptotic cells counted. (B) The apoptotic index (mean of three independent experiments \pm $\mathrm{SE})$ is shown. Values are significant $\mathrm{a}(\mathrm{p}<0.05), \mathrm{b}(\mathrm{p}<0.005)$ and $\mathrm{c}(\mathrm{p}<0.0005)$ with respect to the cells treated with IM alone.
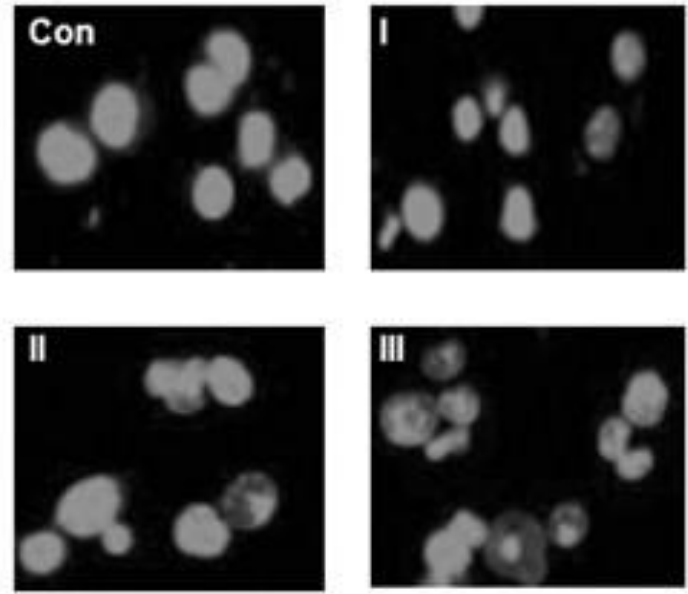

A

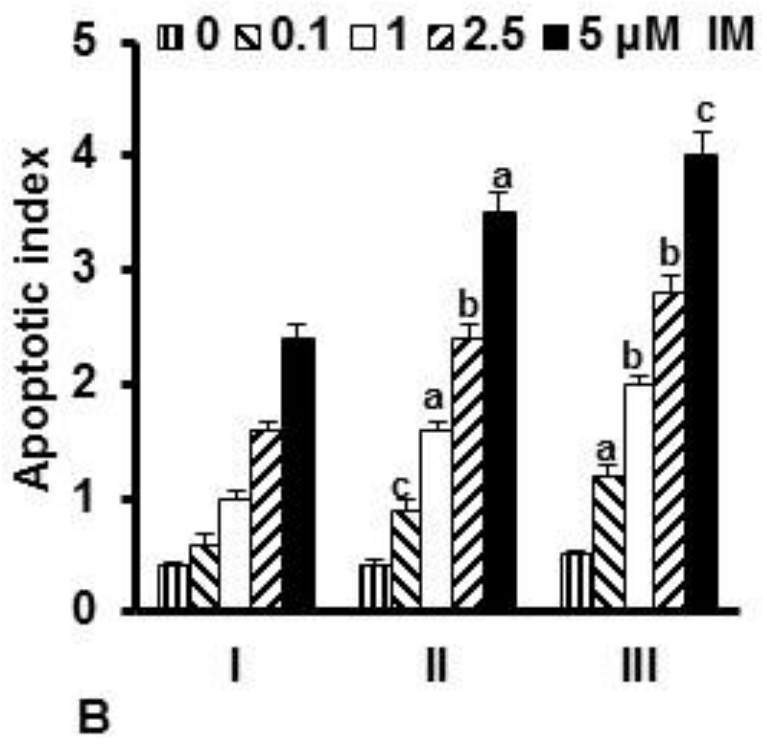


Fig.6 Effect of IM on apoptosis as influenced by Curcumin. Cells treated with IM(I) and with curcumin, simultaneously(II) and pretreatment(III) were studied for apoptosis. (A) Fluorimetric analysis reveal increased activities of caspases in all the treatment modalities, effect is highest in III. Values are significant $a(p<0.05), b(p<0.005)$ and $c(p<0.0005)$ with respect to IM control. (B) DNA fragmentation, hallmark of apoptosis after gel electrophoresis. (C) Representative micrographs showing PARP degradation. (Con) shows untreated cells, (I), (II), (III) respectively represent cells after treatment with $5 \mu \mathrm{M}$ IM alone, IM with Curcumin, simultaneously \& pretreated.

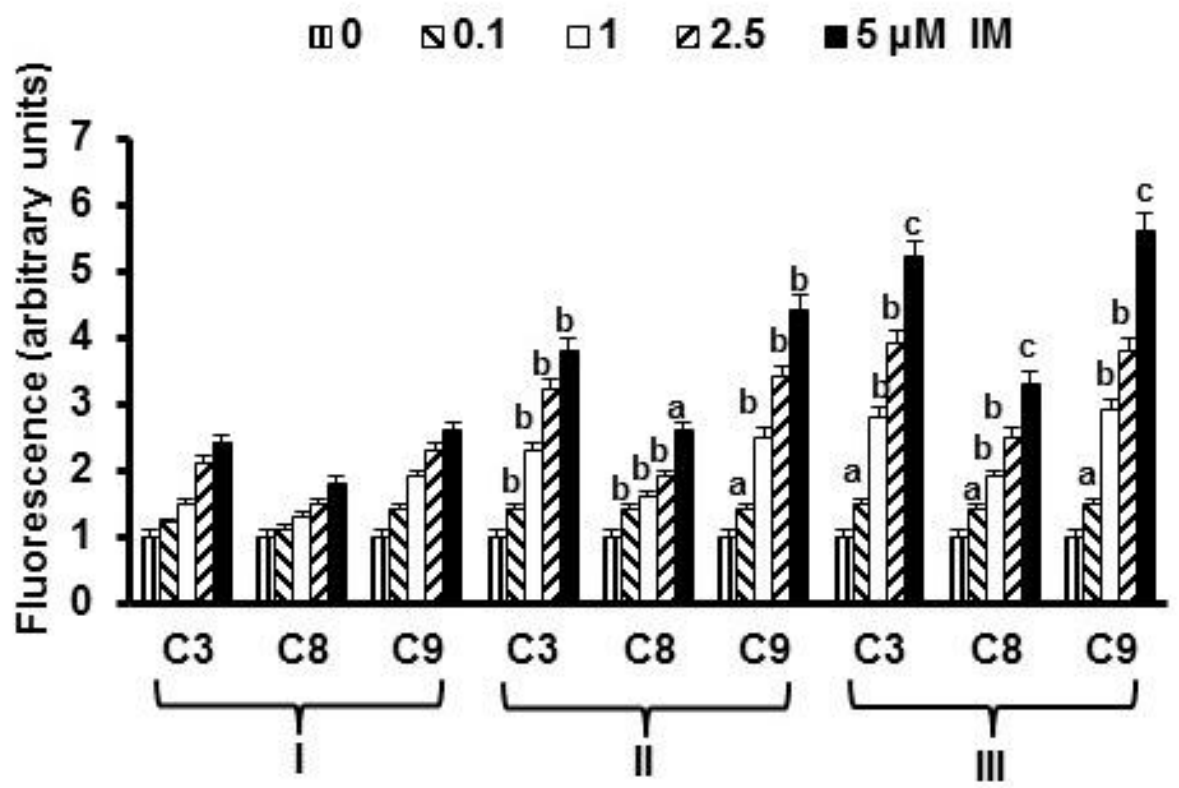

A
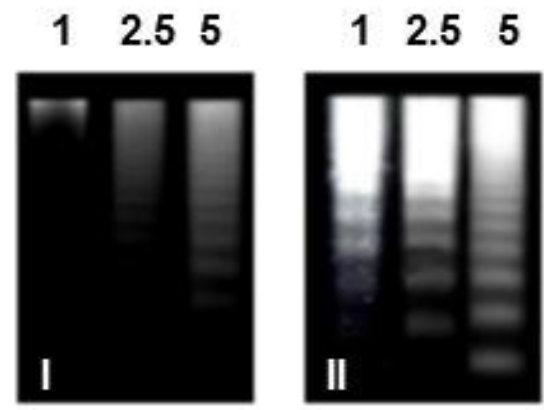

$12.5 \quad 5 \mu \mathrm{M}$ IM

B
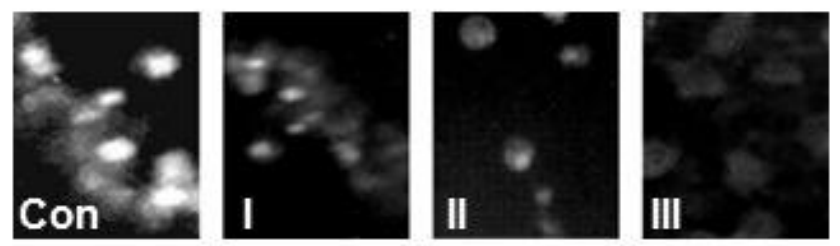

C

Elevated expression of tumor markers like cytokines (TNF- $\alpha$, IL-8), NF- $\kappa \mathrm{B}$ (p50 and p65), survivin, p210 ${ }^{\mathrm{Bcr}-\mathrm{Abl}}$ and HSP 90 and diminished level of anti-inflammatory cytokine IL-10 needs to be earmarked to control leukemogenesis. Present findings 
support the notion that proteins mentioned in the study are involved in development of leukemia, and hence their modulation by a combination of IM and curcumin may ultimately lead to cell killing. Activation of caspases $3 \& 9$ are more than caspase 8, indicating that intrinsic pathway of apoptosis is mainly responsible for death of leukemia cells by this combinatorial treatment modality. Activation of caspases leads to degradation of an important protein poly ADP ribose polymerase (PARP), which is more pronounced in presence of curcumin. Previous reports from our laboratory indicated enhancement of efficacy of IM by curcumin. Among several treatment modalities, best result was obtained when the cells were pre-treated with curcumin prior to IM administration (Sarkar et al., 2014). Present study aims to elucidate the detailed mechanism behind the chemo-enhancement of IM by curcumin. Present study suggests that curcumin is capable of augmenting the efficiency of IM through modulation of cytokines and associated proteins. The combinatorial treatment showed better efficacy in modulating biomarkers than the drug alone. Prior treatment with curcumin induces higher extent of apoptosis than IM alone as revealed by increased apoptotic index, activation of caspases, DNA ladder formation and PARP degradation.

In a nut shell, IM efficiently modulated the aberrant expression of the proteins, but the concentration required to get the desired effect is associated with severe toxicity. Presence of curcumin along with IM comes to the rescue as similar effect could be achieved at a much lower concentration of drug IM. It was reported that curcumin (Reuter et al., 2009) and IM (Zhang et al., 2014; Ciarcia et al., 2012) individually are capable to regulate the expression of these proteins. Present study emphasized the synergistic effect of curcumin and IM in leukemia therapy. Sensitizing the cancer cells with curcumin, prior to drug administration could be a better modality. This is in accordance with other studies which show that phytochemicals act as chemo-enhancers (Doughari et al., 2009). Therefore, in order to tackle the severe side effects of conventional anti-cancer drugs, adjuvant therapy could be a helpful effective modality. Further research is warranted to establish the role of curcumin in combinatorial treatment in leukemia.

\section{Acknowledgement}

Authors are indebted to DST for partial funding of the project. Thanks are due to Director, CNCI for providing infrastructural facilities.

\section{References}

Ahmeda, M.B., H.H. Shehataa, M. Moussaa, and Ibrahim T.M. 2012. Prognostic significance of survivin and tumor necrosis factor-alpha in adult acute lymphoblastic leukemia. Clin Biochem., 45(1-2): 112-116.

An, X., A.K. Tiwari, Y. Sun, P.R. Ding, C.R. Ashby JR, and Chen, Z.S. 2010. BCRABL tyrosine kinase inhibitors in the treatment of Philadelphia chromosome positive chronic myeloid leukemia: a review. Leuk. Res., 34(10): 1255-1268.

Anand, M.S., N. Varma, S. Varma, K.S. Rana, and Malhotra, P. 2012. Cytogenetic \& molecular analyses in adult chronic myelogenous leukaemia patients in north India. Indian J Med Res., 135, 4248.

Ciarcia, R., M.T. Vitiello, M. Galdiero, C. Pacilio, V. Iovane, D. d'Angelo, D. Pagnini, G. Caparrotti, D. Conti, V. Tomei, S. Florio, and Giordano, A. 2012. Imatinib treatment inhibit IL-6, IL-8, NF-KB and AP-1 production and modulate intracellular calcium in CML 
patients. J. Cell Physiol., 227 (6): 27982803.

Doughari, J.H., I.S. Human, S. Bennade, and Ndakidemi, P.A. 2009. Phytochemicals as chemotherapeutic agents and antioxidants: Possible solution to the control of antibiotic resistant verocytotoxin producing bacteria. $J$. Med. Plants Res., 3(11), 839-848.

Driessler, F., K. Venstrom, R. Sabat, K. Asadullah, and Schottelius, A.J. 2004. Molecular mechanisms of interleukin10-mediated inhibition of $\mathrm{NF}-\kappa \mathrm{B}$ activity: a role for p50. Clin. Exp. Immunol., 135(1), 64-73.

Elliott, C.L., V.C. Allport, J.A. Loudon, G.D. $\mathrm{Wu}$, and Bennett, P.R. 2001. Nuclear factor-kappa B is essential for upregulation of interleukin-8 expression in human amnion and cervical epithelial cells. Mol. Hum. Reprod., 7(8), 787790.

Flandrin, P., D. Guyotat, A. Duval, J. Cornillon, E. Tavernier, N. Nadal, and Campos, L. 2008. Significance of heatshock protein (hsp) 90 expression in acute myeloid leukemia cells. Cell Stress Chaperones.,13(3): 357-364.

Gopalakrishnan, A., and Tony kong, A.N. 2008. Anticarcinogenesis by dietary phytochemicals: cytoprotection by $\mathrm{Nrf} 2$ in normal cells and cytotoxicity by modulation of transcription factors NFkappa B and AP-1 in abnormal cancer cells. Food Chem. Toxicol., 46(4), 12571270.

Henkes, M., H. Van der kuip, and Aulitzky W.E. 2008. Therapeutic options for chronic myeloid leukemia: focus on imatinib (Glivec ${ }^{\circledR}$, Gleevec ${ }^{\mathrm{TM}}$ ). Ther Clin. Risk Manag., 4(1), 163-187.

Hermann, B.G., and Frischauf, A.M. 1987. Isolation of genomic DNA. Methods Enzymol (US). 152, 180-183.

Jiao, Y., J. Wilkinson, X. Di, W. Wang, H. Hatcher, N.D. Kock, R. D'Agostino Jr, M.A. Knovich, F.M. Torti, and Torti, S.V. 2009. Curcumin, a cancer chemopreventive and chemotherapeutic agent, is a biologically active iron chelator. Blood, 113(2): 462-69.

Kelly, R.J., A. Lopez-Chavez, D. Citrin, J.E. Janik, and Morris J.C. 2011. Impacting tumor cell-fate by targeting the inhibitor of apoptosis protein survivin. Mol. Cancer, 10, 35-45.

Lawrence, T. 2009. The Nuclear Factor NF$\kappa \mathrm{B}$ Pathway in Inflammation. Cold Spring Harb Perspect Biol., 1(6), a001651.

Lewis J, A. Devin, A. Miller, Y. Lin, Y. Rodriguez, L. Neckers, and Liu, G. 2000. Disruption of hsp90 function results in degradation of the death domain kinase, receptor-interacting protein (RIP), and blockage of tumor necrosis factor-induced nuclear factorkappaB activation. J. Biol. Chem., 275 (14): 10519-10526.

Magee, M.S., A.E. Snook, G.P. Marszalowicz, and Waldman, S.A. 2013. Immunotherapeutic strategies to target prognostic and predictive markers of cancer. Biomark Med., 7(1), 23-35.

Mughal, T.I., and Schrieber, A. 2010. Principal long-term adverse effects of imatinib in patients with chronic myeloid leukemia in chronic phase. Biologics, 4, 315-323.

Nahleh, Z., A. Tfayli , A. Najm , A. El Sayed, and Nahle, Z. 2012. Heat shock proteins in cancer: targeting the 'chaperones'. Future Med. Chem., 4(7), 927-935.

Naidu, M.U., G.V. Ramana, P.U. Rani, I.K. Mohan, A. Suman, and Roy, P. 2004. Chemotherapy-Induced and/or Radiation Therapy-Induced Oral Mucositis-Complicating the Treatment of Cancer. Neoplasia, 6(5), 423-431.

Reuter, S., J. Charlet, T. Juncker, M.H. Teiten, M. Dicato, and Diederich, M. 2009. Effect of curcumin on nuclear factor kappaB signaling pathways in human chronic myelogenous K562 leukemia cells. Ann. N.Y. Acad. Sci., 1171, 436447. 
Roy, M., R. Sarkar, A. Mukherjee, and Mukherjee, S. 2015. Inhibition of crosstalk between Bcr-Abl and PKC signaling by PEITC, augments imatinib sensitivity in chronic myelogenous leukemia cells. Chem. Biol. Interact., 242,195-201.

Sak, K. 2012. Chemotherapy and Dietary Phytochemical Agents. Chemother. Res. Pract., 2012, 1-11.

Sarkar, R., A. Mukherjee, R. Biswas, J. Biswas, and Roy M. 2014. Sulphoraphane, by virtue of its antioxidant potential down-regulates HSP90 in leukemia cells. Int. J. Curr. Microbiol. App. Sci., 3(1): 476-486.

Sarkar, R., A. Mukherjee, S. Mukherjee, R. Biswas, J. Biswas, and Roy, M. 2014. Curcumin augments the efficacy of antitumor drugs used in leukemia by modulation of heat shock proteins via HDAC6. J. Environ. Pathol. Toxicol. Oncol., 33(3), 247-263.

Sarkar, R., S. Mukherjee, and Roy, M. 2013. Targeting heat shock proteins by phenethyl isothiocyanate results in cellcycle arrest and apoptosis of human breast cancer cells. Nutr. Cancer, 65(3), 480-493.
Singh, Y., M. Palombo, and Sinko, P.J. 2008. Recent Trends in Targeted Anticancer Prodrug and Conjugate Design. Curr. Med. Chem., 15(18), 1802-1826.

Suresh, D., and Srinivasan, K. 2010. Tissue distribution \& elimination of capsaicin, piperine \& curcumin following oral intake in rats. Indian J. Med. Res., 131, 682-691.

Tuorkey, M.J. 2014. Curcumin a potent cancer preventive agent: Mechanisms of cancer cell killing. Interv. Med. Appl. Sci., 6(4), 139-146.

Udensi, U.K., and Tchounwou, P.B. 2014. Dual effect of oxidative stress on leukemia cancer induction and treatment. J. Exp. Clin. Cancer Res., 33, 106-121.

Valent, P. 2007. Imatinib-resistant chronic myeloid leukemia (CML): Current concepts on pathogenesis and new emerging pharmacologic approaches. Biologics, 1(4), 433-448.

Wang, X., and Lin, Y. 2008. Tumor necrosis factor and cancer, buddies or foes? Acta Pharmacol. Sin., 29(11), 1275-1288.

Zhang, L., J.H.L. Fok, and Davies, F.E. 2014. Heat shock proteins in multiple myeloma. Oncotarget, 5(5), 1132-1148.

\section{How to cite this article:}

Mukherjee, A., R. Sarkar, S. Mukherjee, J. Biswas and Roy, M. 2016. Curcumin Boosts up the Efficacy of Imatinib Mesylate in Chronic Myelogenic Leukemia Cell Line K-562 by Modulation of Various Markers. Int.J.Curr.Microbiol.App.Sci. 5(12): 240-255. doi: http://dx.doi.org/10.20546/ijcmas.2016.512.026 